\title{
Atrioventricular block after congenital heart surgery: Analysis from the Pediatric Cardiac Critical Care Consortium
}

Amy J. Romer, MD, ${ }^{\mathrm{a}}$ Sarah Tabbutt, MD, PhD, ${ }^{\mathrm{b}}$ Susan P. Etheridge, MD, ${ }^{\mathrm{c}}$ Peter Fischbach, MD, MA, ${ }^{\mathrm{d}}$ Nancy S. Ghanayem, MD, MS, ${ }^{\mathrm{e}}$ Vadiyala Mohan Reddy, MD, ${ }^{\mathrm{f}}$ Raj Sahulee, DO, ${ }^{\mathrm{g}}$ Ronn E. Tanel, MD, James S. Tweddell, MD, ${ }^{i}$ Michael Gaies, MD, MPH, MS, ${ }^{j}$ Mousumi Banerjee, $\mathrm{PhD},{ }^{k}$ Lauren Retzloff, MPH, ${ }^{\mathrm{j}}$ Wenying Zhang, MS, ${ }^{1}$ and Akash R. Patel, $\mathrm{MD}^{\mathrm{h}}$

\section{ABSTRACT}

Objectives: Our primary aims were to describe the contemporary epidemiology of postoperative high-grade atrioventricular block (AVB), the timing of recovery and permanent pacemaker (PPM) placement, and to determine predictors for development of and recovery from AVB.

Methods: Patients who underwent congenital heart surgery from August 2014 to June 2017 were analyzed for AVB using the Pediatric Cardiac Critical Care Consortium registry. Predictors of AVB with or without PPM were identified using multinomial logistic regression. We used these predictors to model the probability of PPM for the subgroup of patients with intraoperative complete AVB.

Results: We analyzed 15,901 surgical hospitalizations; 422 (2.7\%) were complicated by AVB and $162(1.0 \%)$ patients underwent PPM placement. In patients with transient AVB, 50\% resolved by 2 days, and $94 \%$ resolved by 10 days. In patients who received a PPM, 50\% were placed by 8 days and $62 \%$ were placed by 10 days. Independent risk factors associated with PPM compared with resolution of AVB were longer duration of cardiopulmonary bypass (relative risk ratio, $1.04 ; P=.023$ ) and a high-risk operation (relative risk ratio, $2.59 ; P<.001$ ). Among patients with complete AVB originating in the operating room, those with the highest predicted probability of PPM had a PPM placed only $77 \%$ of the time.

Conclusions: In this cohort, postoperative AVB complicated almost 3\% of congenital heart surgery cases and $1 \%$ of patients underwent PPM placement. Because almost all patients (94\%) with transient AVB had resolution by 10 days, our results suggest there is limited benefit to delaying PPM placement beyond that time frame. (J Thorac Cardiovasc Surg 2019;157:1168-77)

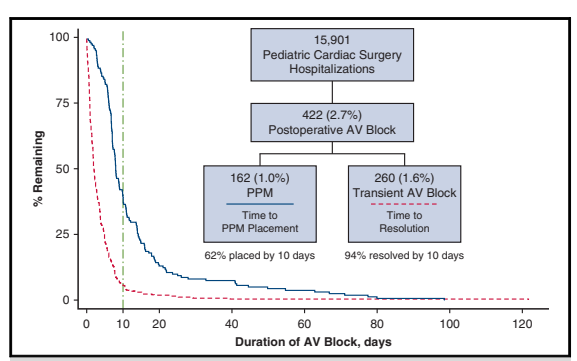

Time to resolution of transient atrioventricular block and permanent pacemaker placement.

\section{Central Message}

Postoperative AVB requiring permanent pacemaker occurred in $1 \%$ of congenital heart surgery patients. Ninety-four percent of patients with transient AVB had resolution by 10 days.

\section{Perspective}

Previous studies on postoperative AVB have been limited by sample size or inadequate data granularity. This large multicenter registry study showed a $1 \%$ incidence of postoperative AVB requiring PPM. Because $94 \%$ of patients with transient AVB had recovered by 10 days, there is likely a limited benefit to delaying PPM placement beyond this time frame.

See Commentaries on pages 1178 and 1180 .
From the ${ }^{\mathrm{a} D e p a r t m e n t}$ of Pediatrics, Division of Cardiology, Stanford University,
Palo Alto, Calif; ${ }^{\mathrm{b}}$ Department of Pediatrics, Division of Critical Care, ${ }^{\mathrm{f}}$ Department
of Surgery, Division of Pediatric Cardiothoracic Surgery, ${ }^{\mathrm{h}}$ Department of Pediat-
rics, Division of Cardiology, University of California, San Francisco, San Fran-
cisco, Calif; ' ${ }^{\mathrm{c}}$ Department of Pediatrics, University of Utah and Primary
Children's Hospital, Salt Lake City, Utah; ${ }^{\mathrm{d}}$ Department of Pediatrics, Sibley Heart
Center, Children's Healthcare of Atlanta, Atlanta, Ga; ${ }^{\mathrm{e}}$ Department of Pediatrics,
Division of Critical Care, Texas Children's Hospital and Baylor College of Medi-
cine, Houston, Tex; ${ }^{\mathrm{g}}$ Department of Pediatrics, Division of Cardiology, New York
University School of Medicine, New York, NY; ${ }^{\mathrm{i}}$ Department of Cardiothoracic
Surgery, Cincinnati Children's Hospital Medical Center, Cincinnati, Ohio; and
${ }^{\mathrm{j} D e p a r t m e n t ~ o f ~ P e d i a t r i c s ~ a n d ~ C o m m u n i c a b l e ~ D i s e a s e, ~ D i v i s i o n ~ o f ~ C a r d i o l o g y, ~}$
C.S. Mott Children's Hospital and University of Michigan Medical School,

${ }^{\mathrm{k}}$ Department of Biostatistics, School of Public Health, and ${ }^{\mathrm{l} C e n t e r}$ for Healthcare Outcomes and Policy, University of Michigan, Ann Arbor, Mich.

This study was supported in part by funding from the University of Michigan Congenital Heart Center,Champs for Mott, and the Michigan Institute for Clinical and Health Research (National Institutes of Health/NCATS UL1TR002240).

Read at the 98th Annual Meeting of The American Association for Thoracic Surgery, San Diego, California, April 28-May 1, 2018.

Received for publication April 29, 2018; revisions received Sept 10, 2018; accepted for publication Sept 30, 2018.

Address for reprints: Amy J. Romer, MD, 750 Welch Rd, Suite 325, Palo Alto, CA 94304 (E-mail: aromer@stanford.edu).

$0022-5223 / \$ 36.00$

Copyright (c) 2018 by The American Association for Thoracic Surgery

https://doi.org/10.1016/j.jtcvs.2018.09.142 

Abbreviations and Acronyms
$\mathrm{AV}=$ atrioventricular
$\mathrm{AVB}=$ atrioventricular block
$\mathrm{CICU}=$ cardiac intensive care unit
$\mathrm{CPB}=$ cardiopulmonary bypass
$\mathrm{ECMO}=$ extracorporeal membrane oxygenation
$\mathrm{PC}^{4}=$ Pediatric Cardiac Critical Care
Consortium
PPM = permanent pacemaker
$\mathrm{RRR}=$ relative risk ratio
STS $=$ Society of Thoracic Surgeons
TGA $=$ transposition of the great arteries
$\mathrm{VSD}=$ ventricular septal defect

or persists at least 7 days after cardiac surgery. ${ }^{8}$ These recommendations are primarily on the basis of small singlecenter studies that have shown that in patients with transient AVB, $80 \%$ recover by 7 days and $>95 \%$ recover by 10 days. ${ }^{1}$ Improved prediction of patients with AVB requiring PPM could potentially identify patients at such high risk that they warrant placement of a PPM during the index operation or after a shorter waiting period.

In this study using a large, multicenter clinical registry, we sought to: (1) describe the contemporary epidemiology and duration of AVB after congenital heart surgery; (2) describe the timing of PPM placement; (3) determine predictors for transient AVB and AVB with PPM; and (4) create a model to predict AVB with PPM on the basis of factors present in the operating room for patients who develop persistent intraoperative complete AVB.

\section{METHODS \\ Data Source}

The Pediatric Cardiac Critical Care Consortium $\left(\mathrm{PC}^{4}\right)$ is a quality improvement collaborative that collects data on all patients with primary cardiac disease admitted to the CICU service at participating hospitals. $\mathrm{PC}^{4}$ maintains a clinical registry to support research and quality improvement initiatives. At the time of this analysis, 25 centers were submitting cases to the $\mathrm{PC}^{4}$ Registry.

Each participating center has a trained data manager who has completed a certification exam. The data managers collect and enter data in accordance with the standardized $\mathrm{PC}^{4}$ Data Definitions Manual. The $\mathrm{PC}^{4}$ registry shares common terminology and definitions with applicable data points from the International Pediatric and Congenital Cardiac Code, Society of Thoracic Surgeons (STS) Congenital Heart Surgery Database, and American College of Cardiology Improving Pediatric and Adult Congenital Treatment Registry, as previously described. ${ }^{9}$ Participating centers are audited on a regular schedule and audit results suggest complete, accurate, and timely submission of data across centers, with the most recent published results showing a major discrepancy rate of $0.6 \%$ across 29,476 fields. ${ }^{10}$ The University of Michigan institutional review board provides oversight for the $\mathrm{PC}^{4}$ Data Coordinating Center; this study was reviewed and approved with waiver of informed consent.

\section{Inclusion and Exclusion Criteria}

We analyzed hospitalizations of patients who underwent an index congenital cardiac operation and recovered immediately postoperatively in the CICU from August 1, 2014 to June 1, 2017 (current version of $\mathrm{PC}^{4}$ with necessary data fields for analysis). Patients were excluded if they had preoperative complete AVB or a preexisting PPM. Patients were also excluded from analysis if they were treated for AVB for fewer than 7 days and died before resolution of AVB.

\section{Data Collection}

For the purposes of this study, postoperative AVB was defined as second- or third-degree AVB that was treated with critical care therapies that started at any time during or after the index operation while in the CICU. Qualifying therapies included temporary pacing, intravenous medications, or a PPM. The start and end times of each episode of AVB receiving 1 of these therapies is recorded in the $\mathrm{PC}^{4}$ registry; this allowed calculation of the exact duration of AVB. AVB did not necessarily occur after the index operation; we also found episodes that occurred after reoperations. We coded all PPM placements during the hospitalization whether placed surgically or in the electrophysiology lab. For the small number of 
cases in which PPM placement was unclear, individual institutions were contacted and chart review was undertaken to ensure no cases were missed.

Other data variables collected include demographic and patient characteristics, diagnoses, comorbidities, operations performed, and outcomes. Each of these variables was recorded for the index operation even if AVB occurred after a subsequent reoperation. We defined a list of highrisk operations a priori by consensus of the investigative team that we believed to be associated with development of AVB on the basis of experience and literature review (Table E1).

\section{Statistical Analysis}

The episode of analysis was the hospitalization, with evaluation of AVB during the CICU admission. Three outcome groups were defined: (1) CICU admissions with no AVB; (2) CICU admissions with AVB that resolved without placement of a PPM, which are referred to as "transient AVB"; and (3) CICU admissions either with AVB and PPM placement or with AVB for $>7$ days that persisted until the time of death, which are referred to as "AVB with PPM." Patients who died after $>7$ days of AVB without resolution were included in the group of AVB with PPM because we assumed these patients would likely have undergone PPM placement if they had survived, on the basis of recommendations from the American College of Cardiology, American Heart Association, and Heart Rhythm Society to place a PPM in patients with AVB without resolution after 7 days. ${ }^{8}$ Descriptive statistics are presented as frequency and percentage for categorical variables and median and interquartile range for continuous variables. After analyzing whether variables were normally distributed, univariate comparisons were performed using the Kruskal-Wallis test for continuous variables and the $\chi^{2}$ or Fisher exact test for categorical data, as appropriate to the distribution. A threshold of $P<.1$ was selected a priori for inclusion of variables from a univariate analysis in a multinomial logistic regression model to identify independent predictors of transient AVB and AVB with PPM placement. We first fit an ordinal logistic regression model, but found the proportional odds assumption to be violated using the Brant test. Therefore, we fitted 2 separate logistic regression models comparing transient AVB with no AVB (reference) and AVB with PPM placement to transient AVB (reference). A hospital-specific random effects term was added to the model to account for clustering of patients within hospitals. Multicollinearity was assessed using the variance inflation factor, and collinear variables were dropped on the basis of clinical judgement. We report the relative risk ratio (RRR) and $95 \%$ confidence interval for each predictor; significant association with the outcome was defined as $P<.05$. A Kaplan-Meier curve was plotted to show time from onset of AVB to either resolution or placement of PPM. We calculated and compared case risk-adjusted mean hospital length of stay and mortality rates across the 3 outcome groups by controlling for patient factors such as age, surgical complexity, comorbid conditions, and STS preoperative risk factors.

We were also interested in determining whether we could predict which patients with complete AVB originating in the operating room would go on to receive a PPM on the basis of patient-related factors present in the operating room. The clinical applicability of such an analysis could allow surgeons to identify patients at such high likelihood of nonresolution that they would consider placing a PPM at the time of initial operation. The patients included in this model were those with complete AVB present on admission to the $\mathrm{CICU}$ from the operating room. We calculated the probability of PPM placement using factors with $P$ value $<.1$ in multivariable logistic regression. We then analyzed the frequency of PPM placement in those with probabilities of $\mathrm{PPM}>.7$ from the model (approximately the top 5\% of all probabilities in the cohort). Analyses were performed using SAS version 9.4 (SAS Institute, Cary, NC) or STATA version 14 (Stata Corp, College Station, Tex).

\section{RESULTS}

A total of 15,901 patients from 25 centers were included in the analysis. Patient and operative characteristics are shown in Table 1 for the overall cohort and within each outcome group. A total of $422(2.7 \%)$ patients had postoperative second- or third-degree AVB requiring treatment; a total of $162(1.0 \%)$ had postoperative AVB and either placement of a PPM $(n=150)$ or died with persistent AVB after at least 7 days of treatment $(n=12)$. Across the 25 centers, the unadjusted incidence of AVB ranged from $0 \%$ to $6.3 \%$, and frequency of PPM placement ranged from $0 \%$ to $3.1 \%$.

\section{Incidence of AVB and PPM According to Type of Operation}

The highest rates of AVB and AVB with PPM were in patients who underwent the Nikaidoh procedure, congenital corrected TGA repair, VSD creation and enlargement, and tricuspid valve replacement. The operations with the highest rates of AVB and PPM are listed in Table 2.

\section{Timing of AVB Resolution and PPM Placement}

In patients with transient AVB, the median time to resolution was 1.9 days (interquartile range, 0.82-4.8) after development of AVB. By 7 days, $86 \%$ had resolved and by 10 days, $94 \%$ had resolved. In patients with AVB with PPM, median time to PPM placement was 8 days (interquartile range, 6.1-14.2), with $62 \%$ placed by 10 days (Figure 1).

Of the patients with complete AVB originating in the operating room, $45.3 \%$ underwent PPM placement.

\section{Predictors of Transient AVB and AVB With PPM}

Preoperative and intraoperative factors associated with transient AVB and AVB with PPM in univariate analysis were under- or overweight compared with normal body weight as defined by the Centers for Disease Control and World Health Organization criteria, any chromosomal abnormality, any syndrome, heterotaxy, extracardiac anomalies, use of cardiopulmonary bypass (CPB), duration of CPB, duration of aortic cross-clamp, STS-European Association for Cardio-Thoracic Surgery risk category, ${ }^{11}$ preoperative or intraoperative extracorporeal membrane oxygenation (ECMO), preoperative hypothyroidism, preoperative endocarditis, and exposure to a high-risk operation.

For patients with any syndrome $121(3.7 \%)$ had AVB and $49(1.5 \%)$ had a PPM placed. For patients with heterotaxy $15(3.3 \%)$ had AVB and $10(2.2 \%)$ had a PPM placed. For patients with the highest STS-European Association for Cardio-Thoracic Surgery risk categories of 4 and 5, 165 $(3.9 \%)$ had AVB and $69(1.6 \%)$ had a PPM placed. For the 6463 patients who underwent at least 1 high-risk operation, $322(5.0 \%)$ had AVB and $135(2.1 \%)$ had AVB with a PPM (Table 1). 
TABLE 1. Preoperative and perioperative characteristics overall and according to outcome

\begin{tabular}{|c|c|c|c|c|c|}
\hline Variable & $\begin{array}{l}\text { All patients } \\
(\mathrm{n}=15,901)\end{array}$ & $\begin{array}{c}\text { No AVB } * \\
(n=15,479)\end{array}$ & $\begin{array}{l}\text { Transient AVB } \\
\quad(n=260)\end{array}$ & $\begin{array}{l}\text { AVB with PPM } \\
\quad(n=162)\end{array}$ & $P$ value \\
\hline \multicolumn{6}{|l|}{ Demographic characteristic } \\
\hline Age group at surgery & & & & & .26 \\
\hline $\begin{array}{l}\text { Preterm neonate }(<30 \text { days old and } \\
<37 \text { weeks' gestation) }\end{array}$ & $422(2.7)$ & $413(2.7)$ & $5(1.9)$ & $4(2.5)$ & \\
\hline $\begin{array}{l}\text { Neonate ( }<30 \text { days old and } \geq 37 \text { weeks' } \\
\text { gestation) }\end{array}$ & $2725(17.1)$ & $2652(17.1)$ & $38(14.6)$ & 35 (21.6) & \\
\hline Infant (30 days to 1 year) & $5733(36.1)$ & 5563 (35.9) & $111(42.7)$ & $59(36.4)$ & \\
\hline Child (1 year to 18 years) & $6308(39.7)$ & $6159(39.8)$ & $95(36.5)$ & $54(33.3)$ & \\
\hline Adult (18 years or older) & $713(4.5)$ & $692(4.5)$ & $11(4.2)$ & $10(6.2)$ & \\
\hline Race & & & & & .27 \\
\hline Hispanic, any race & $2595(16.3)$ & $2532(16.4)$ & $39(15.0)$ & $24(14.8)$ & \\
\hline Caucasian & $8970(56.4)$ & $8718(56.3)$ & $156(60.0)$ & $96(59.3)$ & \\
\hline Black & $2512(15.8)$ & $2456(15.9)$ & $36(13.9)$ & $20(12.4)$ & \\
\hline Asian & $559(3.5)$ & $549(3.6)$ & $5(1.9)$ & $5(3.1)$ & \\
\hline Native American & $38(0.2)$ & $34(0.22)$ & $2(0.77)$ & $2(1.23)$ & \\
\hline Native Pacific Islander & $35(0.2)$ & $34(0.2)$ & $1(0.4)$ & - & \\
\hline Other/multiracial & $897(5.6)$ & $869(5.6)$ & $16(6.2)$ & $12(7.4)$ & \\
\hline Unknown & 295 (1.9) & 287 (1.9) & $5(1.9)$ & $3(1.9)$ & \\
\hline Sex & & & & & .57 \\
\hline Male & $8763(55.1)$ & $8540(55.2)$ & $135(51.9)$ & $88(54.3)$ & \\
\hline Female & $7131(44.9)$ & $6932(44.8)$ & $125(48.1)$ & $74(45.7)$ & \\
\hline \multicolumn{6}{|l|}{ Preoperative factors and diagnoses } \\
\hline Weight, kg & $7.2(4.2-16.9)$ & $7.2(4.2-17.0)$ & $6.5(4.2-15.5)$ & $6.2(4.0-15.5)$ & .41 \\
\hline Weight status at surgery & & & & & .021 \\
\hline Underweight & $3771(23.7)$ & 3648 (23.6) & $81(31.2)$ & $42(25.9)$ & \\
\hline Normal & $11,655(73.3)$ & $11,373(73.5)$ & $170(65.4)$ & $112(69.1)$ & \\
\hline Overweight/obese & $475(3.0)$ & $458(3.0)$ & $9(3.5)$ & $8(5.0)$ & \\
\hline Any specified chromosomal abnormality & $2148(13.5)$ & $2058(13.3)$ & 57 (21.9) & $33(20.4)$ & $<.001$ \\
\hline Any specified syndrome & 3315 (20.9) & 3194 (20.6) & $72(27.7)$ & $49(30.3)$ & $<.001$ \\
\hline Heterotaxy & $457(2.9)$ & $442(2.9)$ & $5(1.9)$ & $10(6.2)$ & .028 \\
\hline Any specified extracardiac anomaly & $2650(16.7)$ & 2561 (16.6) & $57(21.9)$ & $32(19.8)$ & .040 \\
\hline Hypothyroidism & $370(2.3)$ & $355(2.3)$ & $12(4.6)$ & $3(1.9)$ & .044 \\
\hline Pre- or intraoperative ECMO & $350(2.2)$ & $333(2.2)$ & $9(3.5)$ & $8(5.0)$ & .021 \\
\hline $\mathrm{CPR}$ & $178(1.1)$ & $174(1.1)$ & $2(0.8)$ & $2(1.2)$ & .87 \\
\hline Vasoactive infusions at the time of surgery & $928(5.8)$ & $893(5.8)$ & $21(8.1)$ & $14(8.6)$ & .09 \\
\hline Endocarditis & $123(0.8)$ & $116(0.8)$ & $6(2.3)$ & $1(0.6)$ & .034 \\
\hline \multicolumn{6}{|l|}{ Index operation characteristics } \\
\hline $\begin{array}{l}\text { Number of previous cardiothoracic } \\
\text { operations }\end{array}$ & $0(0-1)$ & $0(0-1)$ & $0(0-1)$ & $0(0-1)$ & .76 \\
\hline $\begin{array}{l}\text { Number of previous cardiothoracic } \\
\text { operations according to category }\end{array}$ & & & & & .72 \\
\hline None & $10,799(67.9)$ & $10,518(68.0)$ & $171(65.8)$ & $110(67.9)$ & \\
\hline 1 or 2 & $3988(25.1)$ & $3881(25.1)$ & $70(26.9)$ & $37(22.8)$ & \\
\hline 3 or more & $1114(7.0)$ & $1080(7.0)$ & $19(7.3)$ & $15(9.3)$ & \\
\hline \multicolumn{6}{|l|}{ Surgery type } \\
\hline $\mathrm{CPB}$ & $13,749(86.5)$ & $13,341(86.2)$ & $252(96.9)$ & $156(96.3)$ & $<.001$ \\
\hline No CPB & $2152(13.5)$ & $2138(13.8)$ & $8(3.1)$ & $6(3.7)$ & $<.001$ \\
\hline $\mathrm{CPB}$ time, minutes & $87(50-138)$ & $86(50-136)$ & $122(81.5-187.5)$ & $139.5(86-204)$ & $<.001$ \\
\hline Circulatory arrest & $1486(9.4)$ & $1443(9.3)$ & $22(8.5)$ & $21(13.0)$ & .25 \\
\hline Aortic cross-clamp time, minutes & $41(13-75)$ & $40(13-74)$ & $73.5(44.5-115)$ & $72.5(44-121)$ & $<.001$ \\
\hline STAT risk category & & & & & $<.001$ \\
\hline 1 & $4543(28.6)$ & $4443(28.7)$ & $63(24.2)$ & $37(22.8)$ & \\
\hline 2 & 4837 (30.4) & 4753 (30.7) & $56(21.5)$ & $28(17.3)$ & \\
\hline
\end{tabular}


TABLE 1. Continued

\begin{tabular}{|c|c|c|c|c|c|}
\hline Variable & $\begin{array}{l}\text { All patients } \\
(\mathrm{n}=15,901)\end{array}$ & $\begin{array}{c}\text { No AVB } * \\
(n=15,479)\end{array}$ & $\begin{array}{l}\text { Transient AVB } \\
\quad(\mathbf{n}=\mathbf{2 6 0})\end{array}$ & $\begin{array}{l}\text { AVB with PPM } \\
\quad(n=162)\end{array}$ & $P$ value \\
\hline 3 & $2067(13.0)$ & $1998(12.9)$ & $43(16.5)$ & $26(16.1)$ & \\
\hline 4 & $3553(22.3)$ & $3409(22.0)$ & $87(33.5)$ & $57(35.2)$ & \\
\hline 5 & $666(4.2)$ & $645(4.2)$ & $9(3.5)$ & $12(7.4)$ & \\
\hline Unclassified & $235(1.5)$ & $231(1.5)$ & $2(0.8)$ & $2(1.2)$ & \\
\hline $\begin{array}{l}\text { High-risk operation during or after the index } \\
\text { operation }\end{array}$ & & & & & $<.001$ \\
\hline Surgery, at least 1 high-risk operation $\dagger$ & $6463(40.7)$ & $6141(39.7)$ & 187 (71.9) & $135(83.3)$ & \\
\hline $\begin{array}{l}\text { Catheterization procedure, never high-risk } \\
\text { operation } \dagger\end{array}$ & $1784(11.2)$ & $1767(11.4)$ & $12(4.6)$ & $5(3.1)$ & \\
\hline No high-risk operation or catheterization $\dagger$ & $7654(48.1)$ & $7571(48.9)$ & $61(23.5)$ & $22(13.6)$ & \\
\hline
\end{tabular}

Data are presented as $\mathrm{n}(\%)$ reported for category variables; median (interquartile range) reported for continuous variables. Univariable comparisons were performed using the Kruskal-Wallis test for continuous variables and $\chi^{2}$ or Fisher exact test for categorical data as appropriate. AVB, Atrioventricular block; $P P M$, permanent pacemaker; $C I C U$, cardiac intensive care unit; $E C M O$, extracorporeal membrane oxygenation; $C P R$, cardiopulmonary resuscitation; $C P B$, cardiopulmonary bypass; STAT, Society of Thoracic Surgeons-European Association for Cardio-Thoracic Surgery. *AVB is defined as second- or third-degree AVB occurring in the CICU requiring pharmaceutical or pacing intervention. $\dagger$ At any time before start of AVB or before discharge for those without AVB.

\section{Comparative Analysis of Transient AVB and AVB With a PPM}

In multivariable analysis, comparing patients with AVB with a PPM to those with transient AVB, exposure to at least 1 high-risk operation (RRR, 2.59; $P<.001$ ) and longer duration of CPB (RRR, 1.04; $P=.023$ ) were independently associated with AVB with PPM. The RRR for heterotaxy was high (RRR, 3.76; $P=.069$ ), but the $P$ value fell just short of statistical significance (Table 3 ).

\section{Length of Stay and Mortality}

We conducted a multivariable analysis using linear regression to assess the difference in mean risk-adjusted hospital length of stay between the 3 outcome groups. Covariates included common predictors of length of stay in this population and were on the basis of previous $\mathrm{PC}^{4}$ analyses: age, surgical complexity, preoperative risk factors, and comorbidities. The difference in mean risk-adjusted length of stay relative to patients with no AVB was

TABLE 2. Operations with highest rates of AVB and AVB with PPM*

\begin{tabular}{|c|c|c|c|}
\hline Operation & Number of cases & AVB, $\mathbf{n}(\%) \dagger$ & AVB with PPM, n (\%) \\
\hline Nikaidoh procedure & 19 & $5(26.3)$ & $3(15.8)$ \\
\hline Congenitally-corrected TGA repair & 37 & $9(24.3)$ & $5(13.5)$ \\
\hline VSD creation/enlargement & 74 & $12(16.2)$ & $8(10.8)$ \\
\hline Tricuspid valve replacement & 30 & $4(13.3)$ & $3(10.0)$ \\
\hline Yasui procedure & 12 & $1(8.3)$ & $1(8.3)$ \\
\hline Interrupted aortic arch repair & 33 & $2(6.1)$ & $2(6.1)$ \\
\hline Subvalvular aortic stenosis repair without myectomy & 577 & $54(9.4)$ & $31(5.4)$ \\
\hline Ebstein repair & 76 & $7(9.2)$ & $4(5.3)$ \\
\hline Aortic valvuloplasty & 28 & $2(7.1)$ & $1(3.6)$ \\
\hline Arterial switch procedure, VSD repair, and aortic arch repair & 62 & $7(11.3)$ & $2(3.2)$ \\
\hline Mitral valve valvuloplasty or replacement & 632 & $48(7.6)$ & $19(3.0)$ \\
\hline Coarctation repair and VSD repair & 34 & $3(8.8)$ & $1(2.9)$ \\
\hline Intraventricular tunnel repair & 225 & $19(8.4)$ & $6(2.7)$ \\
\hline VSD repair, multiple & 42 & $6(14.3)$ & $1(2.4)$ \\
\hline Myectomy & 169 & $14(8.3)$ & $4(2.4)$ \\
\hline Complete atrioventricular canal repair & 635 & $44(6.9)$ & $15(2.4)$ \\
\hline Arterial switch procedure and VSD repair & 180 & $10(5.6)$ & $4(2.2)$ \\
\hline VSD repair, patch & 1235 & $37(3.0)$ & $16(1.3)$ \\
\hline Tetralogy of Fallot repair & 891 & $34(3.8)$ & $5(0.6)$ \\
\hline
\end{tabular}

$\overline{A V B}$, Atrioventricular block; PPM, permanent pacemaker; TGA, transposition of the great arteries; VSD, ventricular septal defect. *Operations with fewer than 10 patients excluded. $\nmid \mathrm{AVB}$ is defined as second- or third-degree AV block occurring in the CICU requiring pharmaceutical or pacing intervention. 


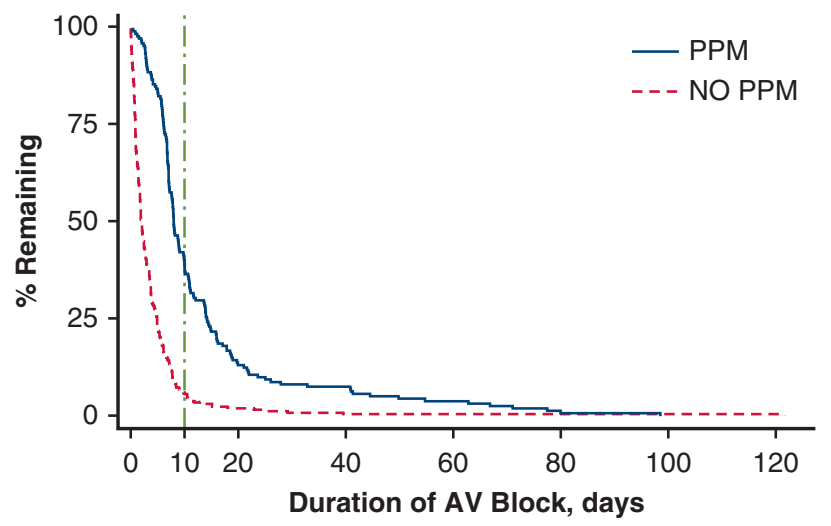

FIGURE 1. Time from onset of atrioventricular $(A V)$ block to permanent pacemaker (PPM) placement (blue solid line)* and time from onset of $\mathrm{AV}$ block to resolution in those with transient AV block (red dashed line). * Patients who died after $>7$ days of unresolved AV block are also included in AV block with PPM group. These patients were censored at time of death.

2.9 days longer for those with transient AVB and 10.7 days longer for those with AVB and a PPM $(P<.001)$.

We then conducted a similar analysis of risk-adjusted mortality within the 3 outcome groups controlling for the same set of covariates as those used in other $\mathrm{PC}^{4}$ mortality risk adjustment models. The risk-adjusted mortality rates were $2.9 \%$ in patients without AVB, $4.7 \%$ in patients with transient AVB, and $9.9 \%$ in patients with AVB and a PPM $(P<.001)$. As in previous analyses, patients who died after 7 or more days of therapy for AVB were included in the AVB with PPM group.

\section{Predicting the Need for PPM Placement During the Index Operation}

Complete AVB originated in the operating room and persisted on admission to the CICU in 247 patients (59\% of all patients with postoperative AVB). Within this subgroup, $112(45.3 \%)$ patients had a PPM placed or died after 7 or more days of persistent AVB. In comparison, 38.4\% of the entire cohort of 422 patients with postoperative AVB had a PPM placed or died after 7 or more days of persistent AVB.

We created a model including factors identified in multivariable analysis to calculate the probability of PPM for patients who developed complete AVB in the operating room. Probabilities of receiving a PPM placement during the hospitalization were calculated using the 3 factors found to be significant in multivariable analysis: heterotaxy, intraoperative ECMO, and whether a high-risk operation was performed. We identified 13 patients with a predicted probability of PPM of $>0.7$, which represented the top $5 \%$ of the cohort. Of this group only $10(77 \%)$ received a PPM.

\section{DISCUSSION}

In this large, multicenter registry study we explored the epidemiology of and risk factors associated with AVB after

TABLE 3. Risk factors for AVB with PPM compared with transient AVB from the logistic regression model

\begin{tabular}{|c|c|c|c|}
\hline Risk factor & RRR & $\begin{array}{l}\text { Normal-based } 95 \% \\
\text { confidence interval }\end{array}$ & $P$ value \\
\hline \multicolumn{4}{|l|}{ Weight group* } \\
\hline Underweight & 0.76 & $0.47-1.22$ & .25 \\
\hline Overweight/obese & 1.60 & $0.43-6.03$ & .49 \\
\hline Any specified chromosomal abnormality & 1.03 & $0.38-2.77$ & .95 \\
\hline Any specified syndrome & 1.01 & $0.42-2.42$ & .99 \\
\hline Heterotaxy & 3.76 & $0.90-15.63$ & .069 \\
\hline Any specified extracardiac anomaly & 0.81 & $0.56-1.15$ & .23 \\
\hline Cardiopulmonary bypass time, $10 \mathrm{~min}$ & 1.04 & $1.01-1.07$ & .023 \\
\hline Aortic cross-clamp time, $10 \mathrm{~min}$ & 0.96 & $0.91-1.01$ & .10 \\
\hline STAT category 4 or 5 vs others & 1.08 & $0.62-1.87$ & .78 \\
\hline \multicolumn{4}{|l|}{ Preoperative risk factors for index operation } \\
\hline Hypothyroidism & 0.41 & $0.10-1.68$ & .21 \\
\hline Pre- or intra-operative ECMO & 1.01 & $0.45-2.28$ & .98 \\
\hline Vasoactive infusions at the time of surgery & 1.21 & $0.62-2.35$ & .58 \\
\hline Endocarditis & 0.24 & $0.02-2.38$ & .22 \\
\hline \multicolumn{4}{|c|}{ High-risk operation during or after index operation $\dagger$} \\
\hline Surgery, at least 1 high-risk operation & 2.59 & $1.68-3.98$ & $<.001$ \\
\hline Catheterization, never high-risk operation & 1.04 & $0.37-2.95$ & .94 \\
\hline
\end{tabular}

$R R R$, Relative risk ratio; STAT, Society of Thoracic Surgeons-European Association for Cardio-Thoracic Surgery; ECMO, extracorporeal membrane oxygenation. *Reference group: normal weight. $\nmid$ Reference group: no high-risk operation or catheterization procedure. 
congenital heart surgery in the modern era. Previous studies on postoperative AVB after congenital heart surgery have primarily been single-center studies. ${ }^{1,3-5,12}$ A few multicenter administrative database studies have been performed with limited patient-level granularity. ${ }^{2,7}$ Our analysis is drawn from data collected by a multicenter CICU collaborative and has the benefit of large numbers and significant detail relevant to the timing of AVB and treatments. In addition, $\mathrm{PC}^{4}$ data collection is directly linked to the STS database at participating centers, thus ensuring consistent coding of diagnoses and operations between the 2 registries.

Our finding of a $2.7 \%$ incidence of postoperative AVB and $1 \%$ incidence of AVB with PPM are similar to previous studies. ${ }^{1-5,7}$ The highest incidence of AVB with PPM occurred in patients who underwent the Nikaidoh procedure, congenitally corrected TGA repair, VSD creation or enlargement, and tricuspid valve replacement. There was considerable variation in rates of AVB and PPM placement between participating centers. Comparison between different institutions is difficult to interpret in the absence of risk adjustment, however, each of the participating hospitals in this analysis performs the full spectrum of congenital heart surgery, and is a referral center for complex neonatal repairs. Further risk-adjusted benchmarking of AVB rates across centers could identify those with the lowest and highest rates of AVB and provide insight into opportunities for quality improvement.

Patients who had transient AVB and AVB with a PPM had significantly longer adjusted mean length of stay and higher adjusted mortality compared with patients without AVB. These differences in outcomes are consistent with previously published studies and highlight the morbidity, mortality, and potential for increased incurred cost associated with postoperative AVB. . $, 3,5,7^{-}$

Risk factors independently associated with need for PPM in patients with postoperative AVB were exposure to a highrisk operation and longer $\mathrm{CPB}$ time. The association with a high-risk operation is expected because operations involving tissue in close proximity to the $\mathrm{AV}$ node and conduction system would be expected to have a greater risk than those that occur remote from the conduction system. In addition, patients who undergo certain operations might also have a higher risk of AVB on the basis of their underlying cardiac anatomy, such as in L-looped ventricles for which patients have been shown to have increased risk of AVB even in the absence of surgery. ${ }^{13}$ The association with bypass time might be due to a direct effect of longer bypass time, or might be a surrogate for increased surgical complexity. Heterotaxy might also be a predictor of PPM in those with AVB, which is consistent with an underlying predilection for $\mathrm{AV}$ conduction defects in patients with heterotaxy, especially left atrial isomerism. ${ }^{14}$
In this study we were unable to create a model that accurately predicted which patients with intraoperative complete AVB would go on to need a PPM. Although patients with intraoperative complete AVB had a greater probability of PPM if they underwent a high-risk operation, had intraoperative ECMO, or had heterotaxy, the model had an accuracy level of only $77 \%$ in the patients with the top $5 \%$ predicted probability. This is insufficient to definitively recommend placement of permanent pacing wires or a PPM during an index operation in these patients. Identification and incorporation of additional variables identified in the operating room could enhance the model, potentially leading to a practice change of PPM placement during the index operation or after a shorter waiting period in a specific subset of patients. A larger sample of patients would be necessary to conduct such an analysis.

The timing of resolution of transient AVB in this cohort ( $86 \%$ by 7 days) is similar to previously published reports, and supports the current recommendations by the American College of Cardiology, American Heart Association, and Heart Rhythm Society to consider PPM placement in patients with AVB that persists beyond 7 days. ${ }^{1,8}$ Alternatively, a more conservative threshold of PPM placement at 10 days could also be considered because $94 \%$ have recovered by this time point. However, delaying PPM placement beyond 10 days is not supported by these results. Interestingly, we found that only $62 \%$ of patients had their PPM placed by 10 days. Unfortunately the $\mathrm{PC}^{4}$ registry does not provide definitive insight into the contributors to decision-making around timing of PPM placement. We do not know if delays were because of a desire to wait longer for return of conduction or because of other factors such as patient instability or availability of operating room time that precluded earlier PPM placement. The optimal timing of PPM placement has important implications. There is a challenging balance between reducing hospital length of stay and associated patient-related risks and cost and avoiding placement of a potentially unnecessary device.

\section{Study Limitations}

The limitations of our study include those inherent to any observational database study. There are a number of different factors that might affect the development and recovery of AVB that were not included in the $\mathrm{PC}^{4}$ registry. These include the location of injury within the conduction system, genetic polymorphisms, QRS escape rhythm morphology and rate, the presence and frequency of any underlying $\mathrm{AV}$ nodal conduction during the period of $\mathrm{AVB},{ }^{5,12}$ and the surgeon's intraoperative concern for permanent AVB. Our evaluation is also limited by a lack of detail about decision-making around the timing of PPM placement. Finally, assessment of long-term AV nodal conduction was not included as part of this study, thus patients 
who underwent PPM placement for postoperative AVB and eventually had return of normal sinus rhythm ${ }^{15}$ or patients who developed late-onset AVB after discharge and subsequently required PPM, ${ }^{4}$ were not captured in this study.

\section{CONCLUSIONS}

In summary, we performed an analysis of AVB and PPM placement after congenital heart surgery using the $\mathrm{PC}^{4}$ multicenter registry. AVB with PPM occurred in $1 \%$ of patients. For patients who developed AVB, the risk of receiving a PPM was independently associated with duration of CPB and exposure to a high-risk operation. Resolution of transient AVB occurred by 7 days in $86 \%$ and by 10 days in $94 \%$ of patients, which supports a waiting period of 7 to 10 days postoperatively before PPM placement. Interestingly, only $62 \%$ of PPMs were placed by 10 days. We were unable to create a strong model to predict which patients with intraoperative AVB would benefit from PPM placement during the index operation. Further studies evaluating risk factors associated with need for PPM placement might help elucidate when early PPM placement is appropriate, and which patients deserve a full 10-day waiting period for return of conduction.

\section{Webcast}

You can watch a Webcast of this AATS meeting presentation by going to: https://aats.blob.core.windows.net/ media/18Apr30/24ABC \%20Congenital $\% 20$ SS/S67\%20\%20Part\%201/S67_2_webcast_021726664.mp4.

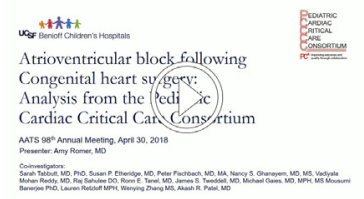

\section{Conflict of Interest Statement}

Authors have nothing to disclose with regard to commercial support.

We acknowledge the data collection teams at all of the participating centers.

\section{References}

1. Weindling SN, Saul JP, Gamble WJ, Mayer JE, Wessel D, Walsh EP. Duration of complete atrioventricular block after congenital heart disease surgery. Am J Cardiol. 1998;82:525-7.

2. Anderson JB, Czosek RJ, Knilans TK, Meganathan K, Heaton P. Postoperative heart block in children with common forms of congenital heart disease: results from the KID Database. J Cardiovasc Electrophysiol. 2012;23:1349-54.

3. Ayyildiz P, Kasar T, Ozturk E, Ozyilmaz I, Tanidir IC, Guzeltas A, et al. Evaluation of permanent or transient complete heart block after open heart surgery for congenital heart disease. Pacing Clin Electrophysiol. 2016;39:160-5.

4. Lin A, Mahle WT, Frias PA, Fischbach PS, Kogon BE, Kanter KR, et al. Early and delayed atrioventricular conduction block after routine surgery for congenital heart disease. J Thorac Cardiovasc Surg. 2010;140:158-60.
5. Murray LE, Smith AH, Flack EC, Crum K, Owen J, Kannankeril PJ. Genotypic and phenotypic predictors of complete heart block and recovery of conduction after surgical repair of congenital heart disease. Heart Rhythm. 2017;14:402-9.

6. Jou CJ, Etheridge SP, Minich LL, Saarel EV, Lambert LM, Kouretas PC, et al. Long-term outcome and risk of heart block after surgical treatment of subaortic stenosis. World J Pediatr Congenit Heart Surg. 2010;1:15-9.

7. Liberman L, Silver ES, Chai PJ, Anderson BR. Incidence and characteristics of heart block after heart surgery in pediatric patients: a multicenter study. J Thorac Cardiovasc Surg. 2016;152:197-202.

8. Epstein AE, DiMarco JP, Ellenbogen KA, Estes NA III, Freedman RA, Gettes LS, et al. ACC/AHA/HRS 2008 guidelines for device-based therapy of cardiac rhythm abnormalities: a report of the American College of Cardiology/ American Heart Association task force on practice guidelines (Writing Committee to revise the ACC/AHA/NASPE 2002 guideline update for Implantation of Cardiac Pacemakers and Antiarrhythmia Devices) developed in collaboration with The American Association for Thoracic Surgery and Society of Thoracic Surgeons [errata in 2009;53:1473, 2009;53:147]. J Am Coll Cardiol. 2008;51: e1-62.

9. Gaies M, Cooper DS, Tabbutt S, Schwartz SM, Ghanayem N, Chanani NK, et al. Collaborative quality improvement in the cardiac intensive care unit: development of the Paediatric Cardiac Critical Care Consortium (PC4). Cardiol Young. 2015;25:951-7.

10. Gaies M, Donohue JE, Willis GM, Kennedy AT, Butcher J, Scheurer MA, et al Data integrity of the Pediatric Cardiac Critical Care Consortium (PC4) clinical registry. Cardiol Young. 2016;26:1090-6.

11. Jacobs JP, Jacobs ML, Maruszewski B, Lacour-Gayet FG, Tchervenkov CI, Tobota Z, et al. Initial application in the EACTS and STS Congenital Heart Surgery Databases of an empirically derived methodology of complexity adjustment to evaluate surgical case mix and results. Eur J Cardiothorac Surg. 2012;42: 775-9; discussion: 779-80.

12. Nishimura RA, Callahan MJ, Holmes DR, Gersh BJ, Driscoll DJ, Trusty JM, et al. Transient atrioventricular block after open-heart surgery for congenital heart disease. Am J Cardiol. 1984;53:198-201.

13. Simmons MA, Rollinson N, Fishberger S, Qin L, Fahey J, Elder RW. Modern incidence of complete heart block in patients with L-looped ventricles: does univentricular status matter? Congenit Heart Dis. 2015;10:E237-42.

14. Loomba RS, Willes RJ, Kovach JR, Anderson RH. Chronic arrhythmias in the setting of heterotaxy: differences between right and left isomerism. Congenit Heart Dis. 2016;11:7-18.

15. van Geldorp IE, Vanagt WY, Vugts G, Willems R, Rega F, Gewillig M, et al. Late recovery of atrioventricular conduction after postsurgical chronic atrioventricular block is not exceptional. J Thorac Cardiovasc Surg. 2013;145:1028-32.

Key Words: congenital heart disease, database, pacemaker, pediatric

\section{Discussion}

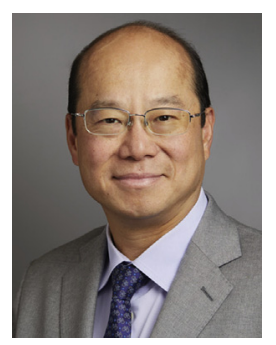

Dr Tain-Yen Hsia (New Haven, Conn). First, I want to congratulate Dr Romer for an outstanding presentation, and I am grateful for the manuscript that you sent to me well ahead of time before the meeting, and I want to thank the association for the privilege to discuss this paper.

So the Google dictionary defines "hope" as a feeling of expectation and a desire for particular things to happen. So for us, or at least for me, when I encounter complete heart block during an operation, hope is all I have got: hope that I didn't nail the AV node with a bundle of His and hope that heart block was just due to some inflammation or irritation of invisible Purkinje fibers, add in a little 
voodoo, steroids, and ICU, and hope lives on. But there are times when hope is helpful and there are times when hope is actually not, because continued patient recovery and progress can be hampered.

So for us, the decision to proceed with permanent pacemaker placement is a balancing act between: (1) a desire to avoid unnecessary pacemaker placement and, (2) a desire to avoid unnecessary delay in placing the pacemaker.

Now it's worth to know, and as Dr Romer mentioned, that the cardiologists got together and recommended that a pacemaker is indicated if heart block persists for at least 7 days after surgery. So what the ACCH and the Heart Rhythm Society are saying is that it's okay to delay a pacemaker for 7 days, but what they are not saying is when the pacemaker should be placed. Is it the eighth day, 12th day, or longer? So this is the question I think the authors hope that this study can answer by interrogating the Pediatric Cardiac Critical Care registry. I won't repeat all the findings that were clearly presented by Dr Romer, but I will highlight the following that I gleaned from the manuscript.

So, (1) when heart block occurs there is a $62 \%$ chance of no need for a pacemaker; (2) if conduction is to return, $94 \%$ will have it by day 10; and (3) if we go by the cardiologists' cutoff and place a pacemaker by day 7 in all patients with heart block, $14 \%$ of those patients who recover actually will have gotten an unnecessary pacemaker, but $50 \%$ who needed one in the end would have gotten one earlier; and (4) the predicted model the authors created was not a very good one.

So I have 2 questions, one, in one aspect in the manuscript you are trying to find out how many patients should get a pacemaker earlier, but I am actually more curious about how many patients actually got one too early. So in the cohort that got a pacemaker, how many were no longer pacemaker dependent at discharge and at 1 year?

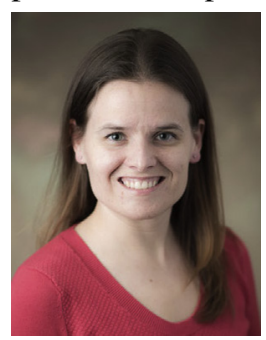

Dr Amy Romer (Stanford, Calif). That's a good question. One thing I did want to clarify is in regard to your statement about the percentage of patients who have AV block who then eventually get a pacemaker. In the entire cohort, only $38 \%$ of patients who had AV block went on to have a pacemaker. However, for the subset that had intraoperative complete heart block, $45 \%$ went on to have a pacemaker.

In regard to your question about which patients are unnecessarily getting pacemakers, that's a great question but those data are not currently available in the PC4 database, but that is something that I think we would like to look at more going forward. The other group that would be interesting to look at are the patients that had long-term transient heart block and evaluate them going forward, what do they look like in 2 years, 5 years? Do they maybe have recurrence later on and they need a permanent pacemaker later.

Dr Hsia. So my second question is that in your discussion you seem to suggest that we have been delaying pacemaker placement in those who wound up needing one, and I would like to highlight that the recommendation is actually not that a pacemaker should be placed on the seventh day but that a pacemaker is indicated after 7 days if the heart block is not expected to resolve. The problem, of course, is that on that seventh day, without a crystal ball, we are still hoping that the conduction tissue would return or the steroid would kick in or something, because we now know that on that day 7 , there is a $14 \%$ chance of recovery.

So on the basis of your study, are you suggesting that day 10 is the absolute cutoff date on which a pacemaker should be placed?

Thank you for a very nice presentation.

Dr Romer. Thank you. In terms of the question of an absolute cutoff day, there are always other factors that might play in but that aren't necessarily in our study, but I think the feeling is that in general with the average patient, intentionally waiting beyond 10 days doesn't necessarily seem to be supported by the data. But of course at that 7-day mark, $14 \%$ of patients is not a small number of patients to say we'll just ignore them and put pacemakers in.

The study brings up a lot of questions, but I am not sure if it provides definitive answers, and I think there is more study to be done in that area.

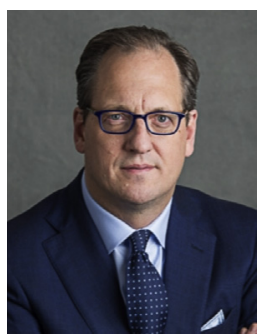

Dr Luca A. Vricella (Baltimore, $M d$ ). Congratulations on a very nice and well presented study. Do you think that it was appropriate at the beginning of your analysis to lump together secondand third-degree heart block? Did you try to sort that out at all?

Dr Romer. It's a good question. What we ended up doing is we did specify patients who had second- and third-degree heart block had to be patients who were treated. So untreated second-degree heart block, basically patients who didn't get medication or pacing, were not included at all. In looking at the intraoperative model, which obviously did not end up being particularly successful, that was one place where we did separate and look at complete heart block independently. While we didn't include degree of heart block as a predictor in our overall model, that's something that we could consider doing. We do see differences between those groups of patients as almost half of those with complete heart block in the OR end up with a pacemaker versus only $38 \%$ in the entire cohort, which is a pretty large difference. 


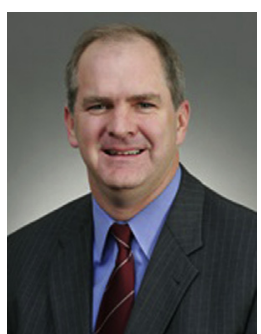

Dr James E. O'Brien (Kansas City, $M o)$. A very nice presentation. If I understood you correctly, a priori by expert consensus you identified highrisk procedures that you thought were at high risk for heart block. Did your data bear that out? So when you looked at the 15,000 , were those procedures that you listed ones that had the higher incidence of heart block and could you share with us what those were?

Dr Romer. Absolutely. Of the entire cohort or 15,000 patients, $40 \%$ had a high-risk procedure. When we look at the patients who had AV block and had a permanent pacemaker, $82 \%$ of them underwent a high-risk procedure. However, there are a number that didn't have a high-risk procedure who developed AV block. Looking through them, some had procedures, such as one is coded as just having a PA reconstruction, which you wouldn't necessarily expect to cause AV block. In these cases the question is, was that because of an issue with coding, was there another procedure as well that we don't know about, or was there something else going on? But in general there was good overlap between what we identified a priori as high-risk procedures and what was found in the study. 
TABLE E1. High-risk operations defined by the investigative team a priori from the $\mathrm{PC}^{4}$ database, data definitions shared with the STS Congenital Heart Surgery Database

\section{$11 / 2$ ventricular repair}

Aortic arch repair and VSD repair

Aortic root translocation over LV (including Nikaidoh procedure)

Aortic stenosis, subvalvular, repair

Aortic stenosis, subvalvular, repair, with myectomy for IHSS

Arrhythmia surgery - atrial, surgical ablation

Arrhythmia surgery - ventricular, surgical ablation

ASO and VSD repair

Arterial switch procedure and VSD repair and aortic arch repair

ASD repair, patch and PAPVC repair

ASD, common atrium (single atrium), septation

Atrial baffle procedure, Mustard or Senning revision

AVC (AVSD) repair, complete

AVC (AVSD) repair, intermediate (transitional)

AVC (AVSD) repair, partial (incomplete)

Cardiac tumor resection

Coarctation repair and VSD repair

Conduit insertion right ventricle to PA and intraventricular tunnel LV to neoaorta and arch reconstruction (Rastelli and Norwood type arch reconstruction, Yasui)

Congenitally corrected TGA repair, atrial switch and ASO (double switch)

Congenitally corrected TGA repair, atrial switch and Rastelli

Congenitally corrected TGA repair, other

Congenitally corrected TGA repair, VSD closure

Congenitally corrected TGA repair, VSD closure and LV to PA conduit

Double outlet LV repair

Double outlet RV, intraventricular tunnel repair

Ebstein repair

HLHS biventricular repair

Konno procedure

LV to aorta tunnel repair

Mitral stenosis, supravalvar mitral ring repair

Mustard

Pulmonary atresia, VSD, MAPCA repair, complete single-stage repair (stage that includes bilateral pulmonary unifocalization and VSD closure and RV to PA connection [with or without conduit])

Pulmonary atresia, VSD, MAPCA repair, status after previous complete unifocalization (includes VSD closure and RV to PA connection [with or without conduit])

Pulmonary atresia, VSD, MAPCA repair, status after previous incomplete unifocalization (includes completion of pulmonary unifocalization and VSD closure and RV to PA connection [with or without conduit])

Pulmonary atresia, VSD (including TOF, pulmonary atresia) repair

Rastelli

REV procedure

Ross-Konno procedure

Senning

Sinus of Valsalva, aneurysm repair

TGA, other procedures (Kawashima, LV-PA conduit, other)

TOF, absent pulmonary valve repair

TOF, AVC (AVSD) repair

TOF repair, no ventriculotomy

TOF repair, RV-PA conduit

TOF repair, ventriculotomy, nontransanular patch

TOF repair, ventriculotomy, transanular patch

Repair of truncus and interrupted aortic arch repair

Truncus arteriosus repair

Valve replacement, common atrioventricular valve

Valve replacement, mitral 


\section{TABLE E1. Continued}

Valve replacement, tricuspid

Valve surgery, other, mitral

Valvuloplasty converted to valve replacement in the same operation, aortic, with Ross-Konno procedure

Valvuloplasty converted to valve replacement in the same operation, common atrioventricular valve

Valvuloplasty converted to valve replacement in the same operation, mitral

Valvuloplasty converted to valve replacement in the same operation, tricuspid

Valvuloplasty, common atrioventricular valve

Valvuloplasty, mitral

Ventricular septation

VSD creation/enlargement

VSD repair, device

VSD repair, patch

VSD repair, primary closure

VSD, multiple, repair

$V S D$, Ventricular septal defect; $L V$, left ventricle; $I H S S$, idiopathic hypertrophic subaortic stenosis; $A S O$, arterial switch operation; $A S D$, atrial septal defect; $P A P V C$, partial anomalous pulmonary venous connection; $A V C$, atrioventricular canal; $A V S D$, atrioventricular septal defect; $P A$, pulmonary artery; $R V$, right ventricle; $T G A$, transposition of the great arteries; HLHS, hypoplastic left heart syndrome; MAPCA, major aortopulmonary collateral artery; TOF, tetralogy of Fallot; REV, réparation à l'étage ventriculaire. 